\title{
MEG Analysis in Alzheimer's Disease Computing Approximate Entropy for Different Frequency Bands
}

\author{
Carlos Gómez*, Student Member, IEEE, Daniel Abásolo, Member, IEEE, Jesús Poza, Member, IEEE, \\ Alberto Fernández, and Roberto Hornero, Member, IEEE,
}

\begin{abstract}
The goal of this study was to analyze the magnetoencephalogram (MEG) background activity in patients with Alzheimer's disease (AD) using a regularity measure: approximate entropy $(A p E n)$. This measure was computed for a broad band $(0.5-40 \mathrm{~Hz})$ as well as typical frequency bands (delta, theta, alpha, beta and gamma). Five minutes of recording were acquired with a 148-channel whole-head magnetometer in 15 patients with probable $A D$ and 15 elderly control subjects. Our results showed that AD patients' MEGs were more regular than controls' recordings at all frequency bands, with the exception of beta. Additionally, there were statistically significant differences $(p<0.01$, Student's $t$-test) at the broad and delta bands. Using receiver operating characteristic curves, the highest accuracy $(83.33 \%)$ was reached at delta band. These results suggest the usefulness of ApEn to gain a better understanding of dynamical processes underlying the MEG recording.
\end{abstract}

\section{INTRODUCTION}

LZHEIMER'S DISEASE
degenerative neurological disorder of unknown etiology that gradually destroys brain cells. It is considered the main cause of dementia in western countries [1]. AD is characterized by neuronal loss and the appearance of neuritic plaques containing amyloid- $\beta$-peptide and neurofibrillary tangles. Clinically, this disease manifests as a slowly progressive impairment of mental functions whose course lasts several years prior to death [2]. Usually, AD starts by destroying neurons in parts of the patient's brain that are responsible for storing and retrieving information. Then, it affects the brain areas involved in language and reasoning. Eventually, many other brain regions are atrophied. Thus, $\mathrm{AD}$ patients may wander, be unable to engage in conversation, appear non-responsive, become helpless and need complete care and attention [3]. Although a definite $\mathrm{AD}$ diagnosis is only possible by necropsy, a differential diagnosis with other types of dementia and with major depression should be attempted. The differential diagnosis includes medical history studies, physical and neurological evaluation, mental status tests, and neuroimaging techniques.

Nowadays, magnetoencephalography (MEG) and

Manuscript received March 30, 2010. This work was supported in part by Ministerio de Ciencia e Innovación under project TEC2008-02241. Asterisk indicates corresponding author.

C. Gómez, D. Abásolo, J. Poza, and R. Hornero are with the Biomedical Engineering Group at Department of Signal Theory and Communications, E.T.S. Ingenieros de Telecomunicación, University of Valladolid, Campus Miguel Delibes, 47011 - Valladolid, Spain (e-mail: cargom@tel.uva.es).

A. Fernández is with the Centro de Magnetoencefalografia Dr. PérezModrego, University Complutense of Madrid, Spain. electroencephalography (EEG) recordings are not used in AD clinical diagnosis. Nevertheless, several studies have demonstrated that the analysis of EEG/MEG recordings could help physicians in the AD diagnosis (for review, see [2] and [4]). EEG and MEG are the only signals that record the synchronous oscillations of cortex pyramidal neurons directly and non-invasively. MEG is a measure of the magnetic brain activity, whereas EEG records the electrical activity of the brain. However, the use of MEG recordings to study the brain background activity offers significant advantages over EEG. The magnetic fields are less distorted by the resistive properties of the skull [5]. Additionally, MEG provides reference-free recordings. Furthermore, the spatial resolution is higher in MEG than in conventional EEG [5]. On the other hand, the magnetic signals generated by the human brain are extremely weak. SQUID (Superconducting QUantum Interference Device) sensors and magnetically shielded rooms are necessary to detect them.

Entropy is a concept addressing randomness and predictability, with greater entropy often associated with more randomness and less system order [6]. Mainly, there are two families of entropy estimators: spectral entropies and embedding entropies [7]. Spectral entropies extract information from the amplitude component of the frequency spectrum. On the other hand, embedding entropies are calculated directly using the time series. This entropies family provides information about how the signal fluctuates with time by comparing the time series with a delayed version of itself [7]. Both spectral and embedding entropies have demonstrated their usefulness in the analysis of EEG/MEG background activity in AD. An increase of entropy values has been found using approximate entropy (ApEn) [6], [8], sample entropy [9], [10], Shannon spectral entropy, Rényi spectral entropy and Tsallis spectral entropy [11].

In this study, we have examined the MEG background activity in patients with probable $\mathrm{AD}$ and in age-matched control subjects using $A p E n$. This entropy measure was computed for a broad band $(0.5-40 \mathrm{~Hz})$ as well as typical frequency bands: delta $(0.5-4 \mathrm{~Hz})$, theta $(4-8 \mathrm{~Hz})$, alpha (8$13 \mathrm{~Hz})$, beta $(13-30 \mathrm{~Hz})$, and gamma $(30-40 \mathrm{~Hz})$. Our purpose was to test the hypothesis that entropy values of the magnetic brain activity would be different in both groups, hence indicating an abnormal type of dynamics associated with AD. 


\section{MATERIALS AND METHODS}

\section{A. MEG recording}

MEGs were recorded using a 148-channel whole-head magnetometer (MAGNES 2500 WH, 4D Neuroimaging) located in a magnetically shielded room. The subjects lay comfortably on a patient bed, in a relaxed state and with their eyes closed. They were asked to stay awake and to avoid eye and head movements. For each subject, five minutes of recording were acquired at a sampling frequency of $678.17 \mathrm{~Hz}$. These recordings were down-sampled by a factor of four, obtaining a sampling rate of $169.55 \mathrm{~Hz}$. Data were digitally filtered between 0.5 and $40 \mathrm{~Hz}$. Finally, artifact-free epochs of 5 seconds (848 samples) were selected and copied as ASCII files to a personal computer for off-line analysis.

\section{B. Subjects}

MEG data were acquired from 30 subjects: 15 patients with probable $\mathrm{AD}$ and 15 elderly control subjects. Cognitive status was screened in both groups with the Mini Mental State Examination (MMSE). The AD group consisted of fifteen patients ( 5 men and 10 women; age $=72.33 \pm 9.04$ years, mean \pm standard deviation, SD) fulfilling the criteria of probable $\mathrm{AD}$, according to the criteria of the Nacional Institute of Neurological and Communicative Disorders and Stroke - Alzheimer's and Related Disorders Association (NINCDS-ADRDA). The mean MMSE score for the patients was $17.67 \pm 3.94$ points. Patients were free of other significant medical, neurological and psychiatric diseases than AD. Moreover, any of the participants in the study used medication that could be expected to influence in the MEG recording.

MEGs were also obtained from fifteen age-matched control subjects without past or present neurological disorders ( 7 men and 8 women; age $=72.53 \pm 5.40$ years, MMSE score $=29.00 \pm 0.33$ points). The local ethics committee approved this study. All control subjects and all caregivers of the patients gave their informed consent for the participation in the current study.

\section{Approximate entropy (ApEn)}

Approximate entropy $(A p E n)$ is a family of statistics that quantifies the signal regularity, notwithstanding its stochastic or deterministic origin [12]. ApEn assigns a nonnegative number to a sequence, with larger values corresponding to greater apparent process randomness or serial irregularity, and smaller values corresponding to more instances of recognizable features or patterns in the data. To compute $A p E n$, two input parameters must be specified: a run length $m$ and a tolerance window $r$. The dimensionless parameter $m$ represents a window length of the number of contiguous time-series points that are compared with one another in forming the ApEn calculation. Briefly, ApEn measures the logarithmic likelihood that runs of patterns that are close (within $r$ ) for $m$ contiguous observations remain close (within the same tolerance width $r$ ) on next incremental comparisons [13]. Comparisons between data sequences must be made with the same values of $m, r$ and $N$, where $N$ is the number of points of the MEG epoch [13]. Pincus has suggested parameter values of $m=1$ or $m=2$, and with $r$ a fixed value between 0.1 to 0.25 times the SD of the original time series [13]. The parameter $r$ is normalized to give $A p E n$ a translation and scale invariance. In this study, ApEn was computed with the established parameters of $m=$ 1 and $r=0.25$ times the SD of the analyzed signal. These parameters provide good statistical reproducibility for sequences longer than $N=60$, as considered herein [12].

The algorithm used to compute the entropy of a signal $\{x(n)\}=x(1), x(2), \ldots, x(N)$ is as follows [12]:

1) Form $m$ vectors $X(1), \ldots, X(N-m+1)$ defined by $X(i)=$ $[x(i), x(i+1), \ldots, x(i+m-1)], i=1, \ldots, N-m+1$. These vectors represent $m$ consecutive values of $x$, starting with the $i$-th point.

2) Define the distance between $X(i)$ and $X(j), d[X(i), X(j)]$ as the maximum absolute difference between their respective scalar components:

$$
d[X(i), X(j)]=\max _{k=1, \ldots, m} \mid x(i+k-1)-x(j+k-1)
$$

3) For a given $X(i)$, let $N_{m}(i)$ denote the number of $j$ ( $j=$ $1, \ldots, N-m+1, j \neq i)$ so that $d[X(i), X(j)] \leq r$. Thus, for $i=1, \ldots, N-m+1$ :

$$
C_{r}^{m}(i)=\frac{N^{m}(i)}{N-m+1}
$$

$C_{r}^{m}(i)$ measure, within a tolerance $r$, the regularity or frequency of patterns similar to a given one of window length $m$.

4) Compute the natural logarithm of each $C_{r}^{m}(i)$ and average it over $i$ :

$$
\varphi^{m}(r)=\frac{1}{N-m+1} \sum_{i=1}^{N-m+1} \ln C_{r}^{m}(i)
$$

5) Increase the dimension to $m+1$ and repeat previous steps in order to obtain $C_{r}^{m+1}(i)$ and $\varphi^{m+1}(r)$.

6) Finally, we define $A p E n$ by:

$$
\operatorname{ApEn}(m, r, N)=\varphi^{m}(r)-\varphi^{m+1}(r)
$$

As $A p E n$ can be applied to short and relatively noisy time series, it has been widely used to extract potentially useful information from biomedical time series such as EEG [6], electrocardiogram [14], concentration time series [15] and respiratory recordings [16], among others.

\section{RESULTS}

ApEn algorithm was applied to all 148 MEG channels to measure the signal regularity. To simplify the analyses, then the results were averaged over all channels. Although both $m$ and $r$ are critical parameters in the performance of $A p E n$, there are no guidelines for optimizing their values. To avoid that noise may bias the ApEn estimation, $r$ must be higher than most of the signal noise [12]. Nevertheless, for small $r$ values, the entropy estimation might fail. Additionally, the accuracy and confidence of the ApEn estimation improve as the number of matches of length $m$ and $m+1$ increases. This 
can be achieved by choosing small $m$ and large $r$. These guidelines lead to use $r$ values between 0.1 and 0.25 times the SD of the time series and $m$ values of 1 or 2 [12]. In this study, we have chosen $m=1$ and $r=0.25$ times the SD of the original time series. The average $A p E n$ values for $\mathrm{AD}$ and control groups at each frequency band are showed in Table I. Our results showed that ApEn values were higher in the control group than in the AD group for all frequency bands, with the exception of beta band. These results suggest that $\mathrm{AD}$ is accompanied by a MEG regularity increase. The differences were statistically significant at delta and broad frequency bands ( $p$-value $<0.01$; Student's $t$-test).

Finally, receiver operating characteristic (ROC) curves were used to assess the ability of these measures to discriminate $\mathrm{AD}$ patients from controls. This method summarizes the performance of a two-class classifier across the range of possible thresholds. The area under the ROC curve (AROC) can be interpreted as the probability that a randomly chosen $\mathrm{AD}$ patient has an entropy value lower than a control subject selected by chance. Fig. 1 represents the ROC curves obtained at each frequency band. The highest accuracy $(83.33 \%)$ and AROC (0.8178) values were achieved at the delta and $0.5-40 \mathrm{~Hz}$ bands, respectively. Table II shows the sensitivity, specificity, accuracy and AROC values obtained with $A p E n$ in each frequency band.

\section{DISCUSSION AND CONCLUSIONS}

We analyzed the MEG background activity from 15 patients with probable $\mathrm{AD}$ and 15 age-matched control subjects by means of a ApEn. Our purpose was to check the hypothesis that MEG background activity was different in AD patients than in control subjects. ApEn has proven to be effective in discriminating $\mathrm{AD}$ patients from controls at the broad and delta bands. Our results revealed that AD patients have lower $A p E n$ values at all frequency bands, with the exception of beta, indicating an increase of the MEG regularity associated with the disease. Our results are in agreement with previous research works that have applied embedding entropies to estimate the regularity of EEG/MEG recordings from $\mathrm{AD}$ patients. In EEG, $A p E n$ values were significantly lower in AD patients at P3 and P4 [6]. With sample entropy, Abásolo et al. [9] found significant differences at parietal and occipital EEG electrodes. These results were subsequently confirmed by MEG studies [8], [10].

Other non-linear methods have been already used to study the MEG activity in AD. Recent studies using a single broad band have suggested a complexity decrease in AD by means of different kinds of complexity measures, as fractal dimension and Lempel-Ziv complexity [8], [10], [17]. Nevertheless, other research works suggested that the differences between AD patients and controls may appear in narrower frequency bands. For instance, Stam et al. [18] found changes of long and short distances interaction in the frequency bands theta, alpha1, beta and gamma using the synchronization likelihood. These changes may reflect loss
TABLE I

MEAN $A P E N$ VALUES For the AD PATIENTS AND the CONTROL SubJECTS AT EACH FREQUENCY BAND

\begin{tabular}{lccc}
\hline \hline & AD group & Control group & $p$-value \\
\hline \hline Broad band & $1.137 \pm 0.233$ & $1.341 \pm 0.119$ & 0.0053 \\
Delta band & $0.184 \pm 0.017$ & $0.198 \pm 0.008$ & 0.0061 \\
Theta band & $0.692 \pm 0.034$ & $0.693 \pm 0.018$ & 0.8897 \\
Alpha band & $1.105 \pm 0.022$ & $1.121 \pm 0.021$ & 0.0562 \\
Beta band & $1.616 \pm 0.049$ & $1.607 \pm 0.065$ & 0.6989 \\
Gamma band & $1.971 \pm 0.017$ & $1.974 \pm 0.009$ & 0.6982 \\
\hline \hline
\end{tabular}

TABLE II

SENSITIVITY, SPECIFICITY, ACCURACY AND AROC VALUES OBTAINED WITH $A P E N$ AT EACH FREQUENCY BAND

\begin{tabular}{lcccc}
\hline \hline & Sensitivity & Specificity & Acurracy & \multirow{2}{*}{ AROC } \\
\hline \hline Broad band & $80.00 \%$ & $80.00 \%$ & $80.00 \%$ & 0.8178 \\
Delta band & $93.33 \%$ & $73.33 \%$ & $83.33 \%$ & 0.8156 \\
Theta band & $60.00 \%$ & $53.33 \%$ & $56.67 \%$ & 0.5511 \\
Alpha band & $66.67 \%$ & $73.33 \%$ & $70.00 \%$ & 0.7133 \\
Beta band & $60.00 \%$ & $53.33 \%$ & $56.67 \%$ & 0.5200 \\
Gamma band & $66.67 \%$ & $53.33 \%$ & $60.00 \%$ & 0.5267 \\
\hline \hline
\end{tabular}

of anatomical connections and/or reduced central cholinergic activity and could underlie part of the cognitive impairment [18]. Using correlation dimension, other study revealed a decreased complexity of the MEG background activity in $\mathrm{AD}$ patients in the low frequency bands, and an increased in the high ones [19].

ROC curves were used to assess the ability of mean $A p E n$ values at different frequency bands to classify $\mathrm{AD}$ patients and control subjects. The highest accuracy was reached at delta band: $83.33 \%$. In previous MEG studies, non-linear methods, together with ROC curves, have been used to distinguish AD patients from control subjects. For instance, an accuracy of $70.7 \%$ was obtained with Lempel-Ziv complexity [8]. In the same study, the accuracy value achieved with ApEn ( $m=2$ and $r=0.25$ times the SD of the time series) was $51.2 \%$, [8]. The highest accuracy in MEG studies was achieved when mean $H F D$ values were analyzed with a ROC curve (87.8\%) [17]. Nevertheless, all these values should be taken with caution due to the small sample sizes.

Our results indicate that entropy measures could be useful in AD diagnosis. Nevertheless, some limitations of our study merit consideration. Firstly, the sample size is small to prove the usefulness of these measures as diagnostic tools. Moreover, the detected decrease in irregularity is not specific to $\mathrm{AD}$, appearing in other brain disorders. Additionally, the results were averaged to simplify the analyses, loosing the spatial information of MEG signals. Finally, our results do not show if $A p E n$ can detect a gradation of the disease process. Future efforts will be focussed to increase the MEG database, as well as to extend the results to other neurodegenerative diseases.

In sum, our study leads us to conclude that MEG background activity in $\mathrm{AD}$ patients is more regular than in elderly control subjects. The results obtained with ApEn 


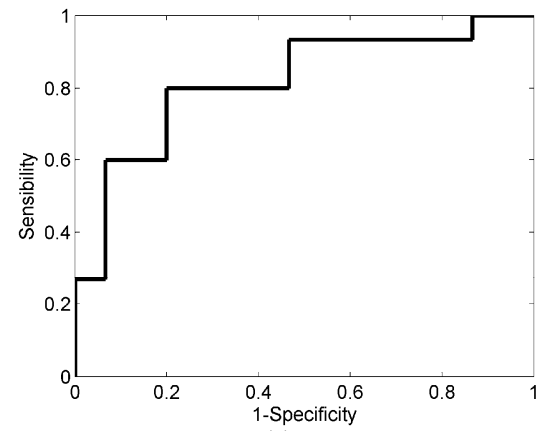

(a)

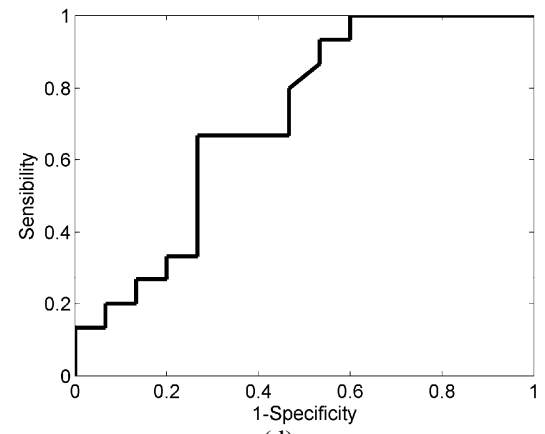

(d)

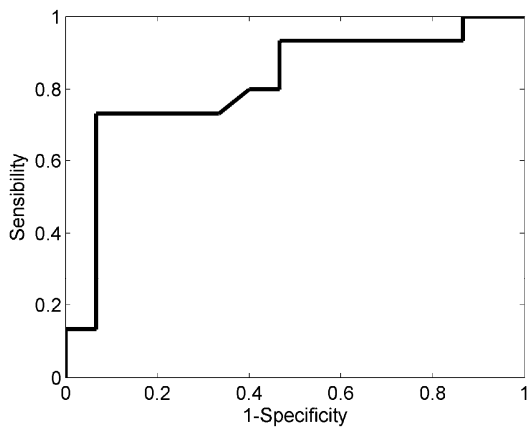

(b)

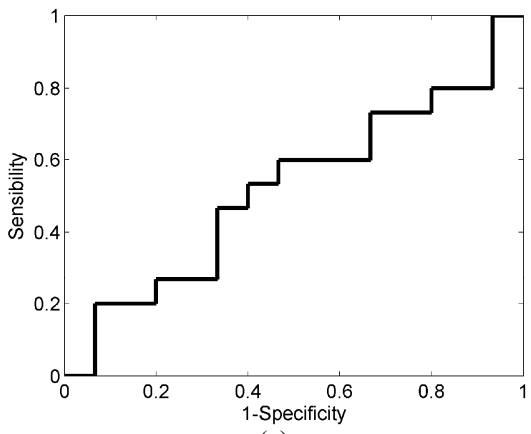

(e)

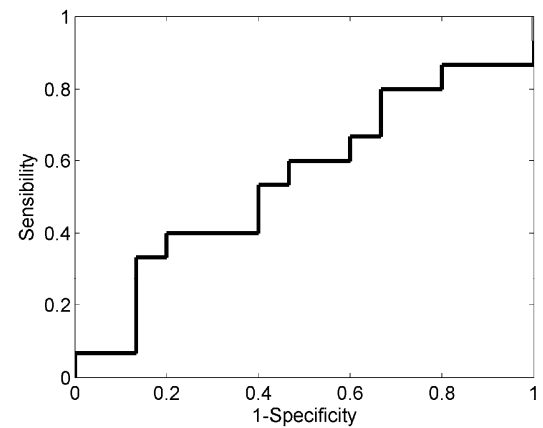

(c)

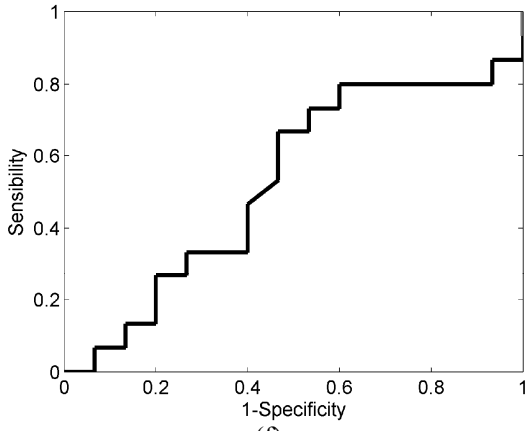

(f)

Fig. 1. ROC curves showing the discrimination between $\mathrm{AD}$ patients and control subjects with $A p E n$ at the following frequency bands: (a) 0.5-40 Hz, (b) delta, (c) theta, (d) alpha, (e) beta, and (f) gamma.

showed significant differences between $\mathrm{AD}$ patients and controls, indicating an abnormal type of dynamics associated with $\mathrm{AD}$. This irregularity reduction seems to be associated with the deficiencies in information processing suffered by $\mathrm{AD}$ patients.

\section{REFERENCES}

[1] T. D. Bird, "Alzheimer's disease and other primary dementias," in Harrison's principles of internal medicine, E. Braunwald, A. S. Fauci, D. L. Kasper, S. L. Hauser, D. L. Longo, and J. L. Jameson, Eds. New York: The McGraw-Hill Companies Inc, 2001, pp. 2391-2399.

[2] J. Jeong, "EEG dynamics in patients with Alzheimer's disease," Clin. Neurophysiol., vol. 115, pp. 1490-1505, 2004.

[3] J. L. Cummings, "Alzheimer's disease," N. Engl. J. Med., vol. 351, pp. 56-67, 2004

[4] C. J. Stam, "Nonlinear dynamical analysis of EEG and MEG: review of an emerging field," Clin. Neurophysiol., vol. 116, pp. 2266-2301, 2005.

[5] R. Hari, "Magnetoencephalography in clinical neurophysiological assessment of human cortical functions," in Electroencephalography: basic principles, clinical applications, and related fields, 5th ed., E. Niedermeyer, and F. Lopes da Silva, Eds. Philadelphia: Lippincontt Williams \& Wilkins, 2005, pp. 1165-1197.

[6] D. Abásolo, R. Hornero, P. Espino, J. Poza, C. I. Sánchez, and R. de la Rosa, "Analysis of regularity in the EEG background activity of Alzheimer's disease patients with approximate entropy," Clin. Neurophysiol., vol. 116, no. 8, pp. 1826-1834, Aug. 2005.

[7] J. W. Sleigh, D. A. Steyn-Ross, C. Grant, and G. Ludbrook, "Cortical entropy changes with general anaesthesia: theory and experiment," Physiol. Meas., vol. 25, no. 4, pp. 921-934, Aug. 2004.

[8] R. Hornero, J. Escudero, A. Fernández, J. Poza, and C. Gómez, "Spectral and non-linear analyses of MEG background activity in patients with Alzheimer's disease," IEEE Trans. Biomed. Eng., vol. 55, pp. 1658-1665, 2008.

[9] D. Abásolo, R. Hornero, P. Espino, D. Álvarez, and J. Poza, "Entropy analysis of the EEG background activity in Alzheimer's disease patients," Physiol. Meas., vol. 27, no. 3, pp 241-253, Mar. 2006.
[10] C. Gómez, R. Hornero, D. Abásolo, A. Fernández, and J. Escudero, "Analysis of MEG background activity in Alzheimer's disease using non-linear methods and ANFIS," Ann. Biomed. Eng., vol. 37, pp. 586594, 2009.

[11] J. Poza, R. Hornero, J. Escudero, A. Fernández, and C. I. Sánchez, "Regional analysis of spontaneous MEG rhythms in patients with Alzheimer's disease using spectral entropies," Ann. Biomed. Eng., vol. 36, pp. 141-152, 2008.

[12] S. M. Pincus, "Approximate entropy as a measure of system complexity," Proc. Natl. Acad. Sci. USA, vol. 88, no. 6, pp. 22972301, Mar. 1991.

[13] S. M. Pincus, "Assesing serial irregularity and its implications for health," Ann. N. Y. Acad. Sci., vol. 954, pp. 245-267, 2001.

[14] V. K. Yeragani, E. Sobolewski, V. C. Jampala, J. Kay, S. Yeragani, and G. Igel, "Fractal dimension and approximate entropy of heart period and heart rate: awake versus sleep differences and methodological issues," Clin. Sci., vol. 95, pp. 295-301, 1998.

[15] O. Schmitz, C. B. Juhl, M. Hollingdal, J. D. Veldhuis, N. Porksen, and S. M. Pincus, "Irregular circulating insulin concentrations in type 2 diabetes mellitus: An inverse relationship between circulating free fatty acid and the disorderliness of an insulin time series in diabetic and healthy individuals," Metabolism, vol. 50, pp. 41-46, 2001.

[16] I. A. Rezek and S. J. Roberts, "Stochastic complexity measures for physiological signal analysis," IEEE Trans. Biomed. Eng., vol. 45, pp. 1186-1191, 1998.

[17] C. Gómez, A. Mediavilla, R. Hornero, D. Abásolo, and A. Fernández, "Use of the Higuchi's fractal dimension for the analysis of MEG recordings from Alzheimer's disease patients," Med. Eng. Phys., vol. 31, pp. 306-313, 2009.

[18] C. J. Stam, B. F. Jones, I. Manshanden, A. M. van Cappellen van Walsum, T. Montez, J. P. A. Verbunt, J. C. de Munck, B. W. van Dijk, H. W. Berendse, and P. Scheltens, "Magnetoencephalographic evaluation of resting-state functional connectivity in Alzheimer's disease," Neuroimage, vol. 32, pp. 1335-1344, 2006.

[19] A. M. van Cappellen van Walsum, Y. A. L. Pijnenburg, H. W. Berendse, B. W. van Dijk, D. L. Knol, Ph. Scheltens, and C. J Stam, "A neural complexity measure applied to MEG data in Alzheimer's disease," Clin. Neurophysiol., vol. 114, pp. 1034-1040, 2003. 